\title{
ANNA LEN
}

DER

\section{CHEMIE und PHARMACIE.}

\author{
LXV. Bandes drittes Heft.
}

Ueber die Zersetzungsproducte des Cyanäthyls durch Einwirkung von Kalium;

von $\boldsymbol{E}$. Frankland und $\boldsymbol{H}$. Kolbe.

In seiner Untersuchung über die Isolirung des Kakodyls *) hebt Bunsen zwei wichtige Momente hervor, welche bei der Abscheidung der organischen Radicale aus ihren liquiden Verbindungen durch Metalle überhaupt wesentlich in Betracht kommen. Die Reduction mufs bei einer niederen Temperatur von Statten gehen, als diejenige ist, bei welcher der zu zersetzende Körper siedet und zweitens darf die sich erzeugende Verbindung des Melalls in dem neu gebildeten Radical nicht unauföslich seyn.

Jene Bemerkungen veranlafsten unš zu einem Versuche über die Abscheidung des Aethyls aus dem Cyanäthyl durch Kalium, da wir als gewifs voraussetzen zu dürfen glaubten, dafs dieses Metall schon bei eiser unter dem Kochpunkte jener Flüssigkeit liegenden Temperatur seine Verwandtschaft zum Cyan äulsern würde.

Ein vorläufiger Versuch erweckte in uns die Hoffnung, unsere Erwartungen in Erfüllung gehen zu sehen. Denn die sehr

-) Annalen der Chemic Bd. XLII. S. 45.

Annal. d. Chemiu u. Pharm. LXV. Bd. 3, Heft. 
energische Einwirkung des Kaliums findet nicht allein schon bei gewöhnlicher Temperatur Statt, sondern das Cyankalium, welches hierbei in reichlicher Menge gebildet wird, löst sich auch in der umgebenden Flüssigkeit so vollständig auf, dafs das Metall bis zu Ende eine blanke Oberfläche behält. Hierbei entwickelt sich von dem Kalium aus ununterbrochen ein brennbares, in Wasser unlösliches Gas mit schwachem ätherartigem Geruch, welches wir anfangs für das gesuchte Aethyl hielten und dessen Untersuchung wir daher unsere Aufmerksamkeit vorzugsweise zuwandten.

Wir bedienten uns zur Darstellung dieses Gases des folgenden Apparates Fig. I. (Siehe die diesem Hefte beigegebene lithographische Tafel.). In die kleine Digerirflasche $A$ von beiläufig 60 Cubiccentimeter Inhalt, auf deren Boden sich vom Steinōl möglichst befreite Stückchen Kalium befinden, mündet durch den doppelt durchbohrten Kork einerseits die Gasleitungsröhre p, anderseits ein bei a zu einer weiten Kugel ausgeblasenes Rohr, dessen unteres umgebogenes Ende zu einer feinen Spitze ausgezogen ist. Der obere Theil desselben ist durch Kaulschuk mit einer mit luftdichtschliefsendem Hahné b versehenen Messingröhre verbunden. Die Kugel a dient als Behälter des Cyanäthyls, welches man nachher durch vorsichtiges Oeffnen des Hahns b tropfenweise auf die Kaliumstücke fliefsen läfst.

Zur Aufnahme des entweichenden Gases ist die in dem Glascylinder CC umgekehrte Glocke B bestimmt, welche unten auf zwei Glasstreifen ruht. Die vom obern Theil der Glocke ausgehende Gasleitungsröhre $g$ ist oberhalb der Krümmung bei $x$ ein wenig ausgezogen und dann durch Kautschuk mit den zur Aufsammlung des Gases für eudiometrische Zwecke bestimmlen weiteren Röhren $e$ und $f$ verbunden. Am hintern Ende befindet sich in der Kautschukröhre $h$ ein massiver Glasstab, durch den ein vollkommner Verschlufs hervergebracht werden kann. 
Kurz vor dem Versuche, ehe dic Gasleitungssröhıre d eingefülort war, wurde in den Cylinder CC frisch ausgekochtes noch warmes Wasser gegossen, durch Saugen mit dem Munile an der Oeffnung bei $h$ das ganze Röhrensystem von $B$ bis h darnit gefüllt, und darauf das offne Ende bei h durch Umbindung des massiven Glasslücks luftdicht verschlossen.

Die ersten Tropfen des Cyanäthyls, welche man durch momentanes Oeffnen des Hahnes b auf das Kalium fallen lä!st, bewirken gewöhnlich eine sehr lebhafte Reaction, welche nicht selten von ciner Feuererscheinung begleitet ist, und die besonders im Anfange sehr rasche Gasentwicklung hat zur Folge, dafs sehr hald alle atmosphärische Luft aus dem Apparate ausgetrieben ist. Erst dann als wir uns versichert hielten, dafs keine Luft mehr darin vorhanden war, wurde die Entwicklungsröhre d unter die Glocke B geführt, die sich dann in dem Mafse mit dem Gase anfüllte, als die Kaliunstücke unter stetigem Zuflurs von Cyanäthyl mehr und mehr verschwanden und sich damit in eine zähe gelbliche Masse verwandelten. Zuletzt mufste die Zerselzung durch gelindes Erwärmen unterstützt werden. Der Versuch wurde unlerbrochen, sobald die Gasentwickelung merklich nachliefs und in der Regel war dann fast alles Kalium consumirt.

Es schien uns zweckmälsig, das in der Glocke angesammelte Gas mehrere Stunden, oder einen Tag lang über dem Wasser stehen zu lassen, damit die darin noch aufgelösten Dämpfe von verdunstetem Cyanälhyl möglichst vollkommen vom Wasser absorbirt würden, wobei wir die Vorsicht gebrauchten, die äufsere Oberfläche desselben mit einer Oelschicht $z u$ bedecken, um der Möglichkeit einer Diffusion des äufsern und innern Gases vorzubeugen. Nachher wurde der Cylinder ganz mit Wasser gefüllt und das Kautschukrohr bei $h$ so lange geöffnet, bis alles Wasser aus dem Röhrensysteme von dem 
unter dem Druck der äufsern Wassersäule stehenden Gase verdrängt war.

Die Kaulschukverbindungen $h$, $s$ und $r$ wurden darauf der Reihe nach, die beiden letzteren doppelt, nit seidner Schnur unterbunden, dicht hinter jeder Unterbindungsstelle durchschnitten und so die Röhren $e$ und $f$ von cinander getrennt. Um letztere vollkominen luftdicht zu machen, lauchten -wir die vier Enden sammt dem Kautschukverschlufs in schinelzendes Wachs ein, was sie Jahre lang gegen jede Verunreinigung durch die äufsere Luft schülzt. Wir zogen diefs Verfahren dem Zuschmelzen mit dem Löthrohr vor, weil wir cine Zersetzung des Gases durch die Erhitzung befürchteten.

Der Resi des Gases wurde zur Bestimmung seines specifischen Gewichtes und zur Verbrennung mit Kupferoxyd verwandt. Zu ersterem Zwecke diente cine 200 Cubikcentimeter fassende gewöhnliche Digerirflasche, deren Hals vor der Glasbläserlampe am Ende verengt war, bis er die Weite eines starken Strohhalmes besafs. Durch einen kleinen eingeschmirgelıen Glasstöpsel konnle sie luftdicht verschlossen werden.

Sie wurde, nachdem mehrere Slückchen geschmolzenen Kulis, zur Entwässerung des später eintretenden feuchten Gases, hineingeworfen und durch Erwärnen an ihreen Boden befestigt waren, mil Quecksilber angefültt, darauf in dem Quecksilber enthaltenden Gefäfse D ungekehrt und mit ihrer Oeffnung über die unter Quecksilber abgebrochene Spitze $\mathrm{x}$ der Rölıre g gebracht. Um den Quecksilberdruck zı überwinden, mufste das in der Glocke B befindliche Gas durch Eingiefsen einer angemessenen Menge Qtiecksilber in den äufseren Cylinder stärker comprimirt werden, bis endlich Gasblasen aus $x$ in die darüber stehende Flasche aufstiegen. Wir füllten dieselbe so weit damit an, bis in dem Halse nur noch cine Quecksilbersäule von elwa 10mm Länge über dem äufseren Niveau hervorragte und brachten sie dann; die Oeffnung unter Quecksilber getaucht, in einen abge- 
schlossenen Raum von conslanter Temperatur, wo wir sie minJestens eine Stunde lang sich überliefsen. Während das Gas die durch ein daneben aufgehängtes Thermometer gemessene Temperatur der umgebenden Luft annahm, wurde es zugleich durch die inwendig befestigten Kalistücke entwässert. Die Höhe ler über dem äufsern Niveau sich erhebenden Quecksilbersäule im Innern des Haises wurde mit einem Zirkel abgemessen und auf einer Millimeterscale abgetragen. Die Flasche wurde hierauf, ohne sie jedoch mit der Hand zu berühren, unter Quecksilber vorsichtig mit dem Stöpsel verschlossen und gewogen, alsdann mit trockner Luft und zuletzt ganz mit Quecksilber gefüllt und beide Mal wieder gewogen.

Wir erhielten dabei folgende Zahlen :

Temperatur wälırend des Verschliefsens . . . 19,70 C. Baromelerstand . . . . . . . . . . . 748,0mm Alzzuziehende Quecksilbersäulc . . . . . . 17,2mm Gewicht des mil Gas gefültten Ballons . . . 53,591 Grm. 'remperatur der Luft in der Wage . . . $20,3^{\circ} \mathrm{C}$. Gewicht des Ballons mit trockner Luft gefült . $53,5775 \mathrm{Grm}$. 'Temperatur beim Wiegen desselben . . . 20,90 $\mathrm{C}$. Capacität des Ballons . . . . . . . . . 210,2 Cbc.

Daraus berechnet sich das specifische Gewicht des Gases zu1 1,075 .

Un das relative Verlällıifs seines Kohlenstoff - und Wasserstoffgehaltes zu ermilteln, leiteten wir ein unbestimmtes Volumen des Gases durch eine mit glühendem Kupferoxyd gefüllte V crbrennungsröhre, mit desem vorderen Ende ein gewogener Chlorcalcium - und Kaliapparal verbunden war. Ein anderes am hinteren Ende der Verbrennungsrölıre angebrachles Chlorcalciumrohr, welches anderseils in die Kaulschukröhre c mündete, mulste das feuchte Gas vor seinem Eintrilt in den Verbrennungsapparat entwässern. Durch behutsames Niederdrücken der Glocke B liefs sich der Gasstrom, dessen Schnelligkeit durch die 
Flüssigkeit des Kaliapparates angezrigt wurde, genau reguliren.

Nachdem sich eine hinlängliche Menge Wasser und Kohlensäure gebildet hatte, wurde die Kautschukröhre c durchschnitten und das im Verbreunungsapparate noch vorhandene Gas durch Saugen am Kaliapparate hindurch gezogen. Bei der Wägung des Chlorcalciumrohrs ergab sich eine Gewichtszunahme von 0,1794 Grm.; das Gewicht des Kaliapparales hatte sich um 0,2915 Grm. vermelort. Das verbrannte Gas enlhielt demnach auf 0,0795 Gewichtstheile Kohlenstoff 0,0199 Theile W'asserstoff, Zahlen, welche genau dem Verhältnifs von $\mathrm{C}: H=2: 3$ entsprechen.

Die eudiometrische Analyse mufste jenes Resultat bestätigen und zugleich über die Condensationsverhältnisse Aufschlufs geben. Wir benutzten dazu je eins der beiden auf die angegebene Weise gefüllten Röhrchen e, f, deren Inhalt unter Quecksilber in das Eudiometer entleert und darin mit Sauerstoff verbrannt wurde. Hinsichtlich des dabei beobachteten Verfalurens und der nöthigen Vorsichtsmaafsregeln verweisen wir auf den Artikel Eudiometrie im chemischen Handwö̀terbuch Bd. II, S. 1050 IT.

I.

beob. Vol. Temp. Queclssilber- Barom. Corr. Vol.

C. stale über d. b. $0^{\circ}$ C.u. im $^{\circ}$ Wanne

\begin{tabular}{|c|c|c|c|c|}
\hline $\begin{array}{l}\text { Vol. d. angewand- } \\
\text { ten Gases (feucht) } / 117,2\end{array}$ & 15,7 & $456,5 \mathrm{~mm}$ & $743,9 \mathrm{~mm}$ & 30,37 \\
\hline $\begin{array}{l}\text { Nach Zulassung von } \\
\text { Sauerstoff (feucht) }\end{array}$ & 15,7 & 216,3 & 743,5 & 169,55 \\
\hline $\begin{array}{l}\text { Nach d.Verbrennung }\{224,5 \\
\text { (feucht) } \cdot \cdot \cdot . \mid\end{array}$ & 16,1 & 286,1 & 743,1 & 94,00 \\
\hline $\left.\begin{array}{l}\text { Nach Absorption der } \\
\text { Kohlensäure(trock.) }\end{array}\right\} 121,6$ & 16,2 & 451,5 & 744,4 & 33,74 \\
\hline $\begin{array}{l}\text { Nach Zulassung von } \\
\text { Wasserstoff (trock.) }\end{array}$ & 16,3 & 241,4 & 744,0 & 153,37 \\
\hline $\begin{array}{l}\text { Nach d.Verbrennung ! } 167,4 \\
\text { (trocken) } . . .\end{array}$ & 16,1 & 404,0 & 744,9 & 53,90 \\
\hline
\end{tabular}


II. beob. Vol, Temp. Quecksilber- Barom. Corr. Vol. C. säule über d. b. $0^{\circ}$ C.u.1m Wanne

Druck

\begin{tabular}{l|lllll}
$\begin{array}{l}\text { Angewandtes Vol. } \\
\text { (feuchl) . . . }\end{array}$ 68,7 & $15,2^{\circ}$ & $500,7 \mathrm{~mm}$ & $744,1 \mathrm{~mm}$ & 15,00
\end{tabular} $\begin{aligned} & \text { Nach Zulassung von } \\ & \text { Sauerstoff (feucht) }\end{aligned}$$\left\{\begin{array}{lllll}331,9 & 15,3 & 229,1 & 743,6 & 157,65\end{array}\right.$ $\begin{aligned} & \text { Nach d. Verbren- } \\ & \text { nung (feucht) }\end{aligned} 281,6 \quad 15,3 \quad 280,9 \quad 744,1 \quad 120,07$ $\begin{array}{lllll}\text { Nach Absorption d. }\{231,6 & 15,3 & 333,1 & 747,7 & 90,92\end{array}$ \begin{tabular}{ll|llll} 
Nach Zulassung v. & v39,0 & 15,4 & 21,5 & 746,8 & 370,05
\end{tabular}

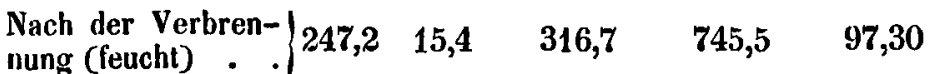

Aus den obigen Analysen berechnet sich, dafs 1 Vol. des Gases nahezu $3 \frac{1}{2}$ Vol. Sauerstoff zur Verbrennung bedarf und 2 Vol. Kohelnsäure erzeugt :

Angewandtes Gasvol. Verbrannler Sauerst. Gebildete $\mathrm{CO}_{2}$

I.

$\begin{array}{rcrrrr} & 30,37 & & 105,60 & & 60,26 \\ = & 1 & : & 3,47 & : & 1,99 \\ & 15,00 & & 51,80 & & 29,15 \\ = & 1 & : & 3,45 & : & 1,94\end{array}$

Fafst man dic obigen Thatsachen zusammen, so ergiebt sich, dafs das fragliche Gas die Zusammensetzung und Condensation des bis jetzt hypothetischen Methyls besitzt. Die in ihren Details bereits mitgetheille Bestimmung seines specifischen Gewichtes hatte die Zahl 1,076 gegeben, welche mit dem berechneten specifischen Gewichte $=1,037$ nahe genug übereinstimmt, wenn man erwägt, dafs dem Gase wahrscheinlich noch ein wenig Cyanäthyldampf beigemengt gewesen ist, wodurch das spec. Gewicht offenbar erhöht werden mufste :

$$
\begin{array}{lll}
1 & \text { Vol. Kohlenstoffdampf }= & 0,82922 \\
3 & \Rightarrow \text { Wasserstoff }= & 0,20730 \\
1 \text { Methylgas } & =1,03652 \\
& &
\end{array}
$$


Das Methyl ist ein farbloses, bei $-18^{\circ}$ nicht condensirbares, in Wasser unlösliches Gas von schwachem ätherartigen Geruch. Alkohol absorbirt davon sein 1,13 faches Volumen. Mit einem gleichen Volumen ausgekochten Alkohol über Quecksilber vermischt und dumit geschültelt, verschwand es bis auf eine kleine Blase, die nicht $1 / 2$ pC, vom Ganzen betrug. Concentrirte Schwefelsäure wirkt nicht auf das Melhyl ein, noch verbindet es sich mit Schwefel oder Jod, wenn man sie in dem Gase erhilzt.

Es war interessant und wichtig zu versuchen, ob sich das Methyl gleich dem Wasserstoff unmittelbar mit Chlor zu Methylchlorür vereinigt. $\mathrm{Zu}$ diesem $\mathrm{Zwecke}$ füllten wir von Neuem einige durch Kautschuk verbundene trockne Röhren gleich denen mit $e$ und $f$ bezeichneten; die vordere derselben war an einem Chlorcalciumrohr und dieses anderseits in die Kautschukröhre c befestigt, welche lelztere während des Füllens der Glocke B in der Mitte unterbunden war. Nachher wurde die Schnur gelöst und durch Niederdrücken der Glocke das Gas durch jenes Röhrensystem hindurchgetrieben. Da das Gasvolumen in der Glocke wohl das Zwanzigfache von der Capaciläl der zu füllenden Rölırchen betrug, so durften wir annehmen, dafs zuletzt alle atmosphärische Luft aus dem Apparat verdrängt war. Die Röhren wurden schliefslich durch Unterbindung der Kautschukverbindungen wie oben verschlossen und von einander getrennt.

Wir fültten darauf ein anderes Röhrchen von derselben Capacilat, wie eins der obigen mit trocknem Clblor und verbanden das eine ausgezogene Ende desselben dann möglichst rasch durch ein starkes Kautschukrohr mit der das Methyl enthaltenden gleichen Röhre, so dafs die beiden Gasc durch jenes mit einander communicirten. Nach 24 stündiger Aufbewahrung an einem dunklen Orte hatien sie sich vollkonmen gemisch, olıne sich jedoch verändert zu haben. Darauf dem zerstreuten 
Licht ausgeselzt, verschwand die Farbe des Chlors mehr und mehr, ein Beweis, dafs nun Vereinigung Statt fand. Nach abcrmals 24 Stunden wurden die beiden Röhren an ihren Enden mit der Lōthrohrflamme ahgeschmolzen, um zu weiteren Versuchen zu dienen.

Beim Wiederöffnen unter Quecksilber zeigte sich, dafs eine bemerkbare Condensalion nicht eingetreten war. Gleichwohl konnte das chlorhallige Product nicht Chlormethyl seyn, da es, wie einige Gasblasen, die man austreten liefs, zu erkennen gaben, eine grofse Menge Salzsäuregas beigemengt enthielt.

Un den Gehalt an Salzsïure quantitativ zl bestimmen, wurde das Gas über Quecksilber in eine kleine in Millimeter eingetheilte und calibrirte trockene Glasgloche übergefüllt und das gemessene Volumen so lange mil einer Kugel von wasserhaltigem phosphorsauren Natron und zuletzt mit einer Kalikugel behandelt, bis die Salzsäure absorbirt war, der Rückstand in ein gröfseres Eudiometer gebracht und mit Sauerstoff verbrannt. Die Analyse gab folgende Zahlen :

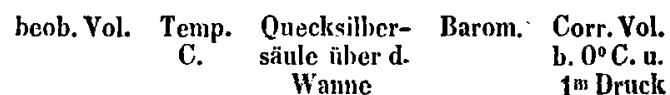

\begin{tabular}{|c|c|c|c|c|c|}
\hline & & & & & \\
\hline $\begin{array}{l}\text { Anfängl. Volumen } \\
\text { (trocken) }\end{array}$ & 3,6 & 18 & $47,2 \mathrm{~mm}$ & $752,5 \mathrm{~mm}$ & 68,35 \\
\hline $\begin{array}{l}\text { Nach Absorption d.) } \\
\text { HE.I (trocken). }\end{array}$ & 55,1 & 18,8 & 95,0 & 755,1 & 34,00 \\
\hline $\begin{array}{l}\text { ullen ! } \\
\text { cht.) ! }\end{array}$ & & 18 & 439,2 & 55,0 & 32 \\
\hline $\begin{array}{l}\text { Nach Zulassung von } \\
0 \text { (feucht). }\end{array}$ & 362,5 & 19,0 & 186,6 & 754,8 & 87 \\
\hline $\begin{array}{l}\text { Verbren-1 } \\
\text { euchit) }\end{array}$ & 281,5 & 19,1 & 69,8 & 753 & \\
\hline $\begin{array}{l}\text { ch Absorption d. } \\
\gamma_{2} \text { (trocken) } .\end{array}$ & 186,1 & 19 & 367,3 & 753 , & \\
\hline $\begin{array}{l}\text { Nach Zulassung von } \\
\text { H (trocken) }\end{array}$ & 462,3 & 20,0 & 86,5 & 752,4 & \\
\hline $\begin{array}{l}\text { Nach der Verbren- } \\
\text { aung (feucht) }\end{array}$ & 240,2 & 20,0 & 311,6 & $\mathbf{7 5 2 , 0}$ & \\
\hline
\end{tabular}

*) Dic Verbrennung war mit einer so heftigen Wïrmeentwieklung verbunden, dals Quecksilloer sich verfü̈htiggte und die lmucuwand des 
Bei der grofsen Schwierigkeit, die das Chlor - und Methylgas enthaltenden Röhren von absolut gleicher Capacität zu erhalten und da es ferner unvermeidlich ist, dafs nicht bejm Zusammenfügen

Eudiometers stellenweise mit einer schwarzen Metallhaut überzog. Gleichzeitig wurde eine grofse Nenge Chlor frei, von dem das rückständige Gas eine tief gelbe Farbe annahm. Es wurde nachher vom Quecksilber yollständig absorbirt, wobei sich der schwarze Beschlag in weifses Quecksilberchlorür verwandelte.

Nach einer früheren Beobachtung von Bunsen (Annalen der Chemie Bd. XLVI, S. 33) soll sich der Wasserstoff bemerkbarer chlorhaltiger Gase durch eudiometrische Verpuffung mit Sauerstoff nicht bestimmen lassen, weil die dabei erzeugte Salzsäure zum Theil unter Ausscheidung von Chlor zersetzt wird. Wenn, im scheinbaren Widerspruch mit dieser Thatsache, bei der obigen Analyse, wie sich aus den Daten derselben ergiebt, eine vollständige Verbrennung des Wasserstoffs und Kohlenstoffs Statt gefunden hat, so liegt der Grund dieser Erscheinung unstreitig in der die Entzündung des Gases begleitenden starken Wärmeentwickelung. Wie sehr die Verbrennlichkeit des Salzsturegases mit der Verbrennungstemperatur zunimmt, geht aus folgenden cudiometrischen Versuchen hervor, in denen Salzsäurcgas mit Knallgas und Sauerstoff in verschiedenen Verhältnissen verbrannt wurde.

\begin{tabular}{|c|c|c|c|c|}
\hline \multicolumn{5}{|c|}{ Versuch $I$. } \\
\hline beob. Vol. & Temp. & $\begin{array}{l}\text { Quecksilber- } \\
\text { sãule über d, } \\
\text { Wanne }\end{array}$ & Bar. & $\begin{array}{l}\text { Corr.Vol b. } \\
0^{\circ} \text { C.u. } 1 \text { in } \\
\text { Druck }\end{array}$ \\
\hline Sauerstotfgas (trocken) 172,3 & $21,2^{\circ}$ & $382,4: \div 1 !$ & $748,9 \mathrm{~mm}$ & 63,1 \\
\hline $\begin{array}{l}\text { Nach Zulassung von } \\
\text { Knallgas (trocken) }\end{array} \mid 285,5$ & 21,2 & 265,9 & 748,0 & 137,6 \\
\hline $\begin{array}{l}\text { Nach Zulassung von } \\
\text { Salzsïuregas (trocken) }\end{array} \mid 362,2$ & 21,2 & 187,4 & 746,9 & 202,7 \\
\hline $\begin{array}{l}\text { Nach der Verbren- }\{237,7 \\
\text { nung (feucht) } \cdot \cdot \cdot\{\end{array}$ & 21,2 & 316,5 & 746,9 & 102,3 \\
\hline $\begin{array}{l}\text { Nach Zulassing von } \\
\text { Wasser (feucht) } \\
.\end{array}$ & 21,2 & $\begin{array}{c}367,0 \\
+ \\
1,5=21 \mathrm{~mm} \\
\text { Wasserdruck. }\end{array}$ & 746,9 & 61,2 \\
\hline
\end{tabular}

Dic Explosion durch den electrischen Funken war im obigen Falle sehr schwach und die geringe Menge des ansgeschiedenen Chlors gab sich kaum durch seine Farbe zu erkennen In dem nächsten Versuche, wo ein kleines Volumen Salzsüure mit einem viel gröfsern Volumen kiailgas und weniger übcrschüssigen Sanerstolf, als zuvor, verbrannt vurcic, war die Entzüindung von einer weit stärkern Wärmentwicke- 
beider kleine Antheile atmosphärischer Luft eingeschlossen twerden, darf man von der vorstehenden Analyse heine absolute Genauigkeit erwarten. Die Resultale gewähren indessen hinreichende Anhaltspunkte, um die atomistische Zusammensetzung des neuen chlorhaltigen Products sicher zu stellen.

Es ergiebt sich daraus zunächst, dafs die Mischung aus gleichen Volumen Salzsäuregas und des andern Products besteht. Die Verbrennung des nach Absorption der Kohlensäure gebliebenen Rückstandes mit Wasserstoff zeigt, dafs dieselhe

lung begleitet. Es fand dabei Sublimation des Quecksilbers und eine reichliche Ausscheidung von Chlor Statt.

$$
\text { Versuch II. }
$$

beob. Vol. Temp. Quecksilber- Bar. Corr.Vol.b.

C. säule über d. $\quad 0^{\circ} \mathrm{C}$. u. $1^{\mathrm{m}}$ Wanne. Druck

\begin{tabular}{|c|c|c|c|c|c|}
\hline Knallgass (trocken) & 305,4 & $21,4^{\circ}$ & $251,2 \mathrm{~mm}$ & $746,8^{\mathrm{nm}}$ & 140,4 \\
\hline $\begin{array}{c}\text { Nach Zulassung von } \\
0 \text { (trocken) }\end{array}$ & 353,0 & 21,4 & 199,8 & 746,2 & 178,8 \\
\hline $\begin{array}{c}\text { Nach Zulassung von } \\
\text { Salzsäureg. (trock.) }\end{array}$ & 405,6 & 21,4 & 146,1 & 745,8 & 225,6 \\
\hline $\begin{array}{c}\text { Nach der Verbren- } \\
\text { nung (feucht) }\end{array}$ & 119,6 & 21,5 & 435,2 & 742,9 & 32,0 \\
\hline $\begin{array}{c}\text { Nach Zulassung von } \\
\text { Wasser (feucht) }\end{array}$ & 111,1 & 21,5 & $\begin{array}{c}419,8 \\
+ \\
=26 \mathrm{~mm} \\
\text { asserdruck }\end{array}$ & 742,9 & $\mathbf{3 1}, \mathbf{3}$ \\
\hline
\end{tabular}

Wenn gleich jene beiden Versuche keine absolute Genauigkeit gewähren, 'weil die letzten Ablesungen über Wasser geschahen, so sind sie doch hinlänglich beweisend fūr die ausgesprochene Behauptung. Bei dem ersten Versuch, in welchem 65 Vol. Salzsăuregas mit 74,5 Vol. Knallgas und 63,1 Vol. Sauerstoff verbrannt wurden, verschwanden vom letzteren nur 1,9 Vol. Es waren also von 65 Vol, Salzsäure nur 7,6 Vol. zerlegt.

Im zweiten Versuch wurden 46,8 Vol. Salzsäure mit 140,4 Vol. Knallgas und 38,4 Vol. Sauerstoff vermischt und hei der Verbrennung 7,1 Vol. Sauerstoff verzehrt. Von 46,8 Vol. Salzsäure blieben demnach nur ' 18,4 Vol. unzersetzt übrig. Im ersten Falle war kaum $1 \%$ Vol., im zweiten über $2 / 3$ Vol. der angewandten Salzsäure verbrannt. 
noch 2,8 Vol. Stickstoff enthielt; demnach sind dem verbrannten Gasvolumen $=32,55$ gegen 3,5 Vol. atmosphärischer Luft beigemengt gewesen, abgesehen von derjenigen Menge des Slickstoffs, die sich bei der Verbrennung zụ Salpetersäure oxydirl, also der Beobachtung entzogen haben mufs. Nach Abzug dieser 3,5 Vol. Luft bleiben 29,0 Vol. des eigentlichen brennbaren Gases, welche der Analyse zu Folge 90,4 Vol. Sauerstoff consumirt und damit 56,0 Vol. Kohlensäure erzeugt haben.

Da, wie der Versuch gezeigt hat, 1 Vol. Methylgas mit 1 Vol. Chlor sich in 1 Vol. Salzsäuregas $(=1 / 2$ Vol. $\mathrm{H}+1 / 2$ Vol.Cl) und 1 Vol. eines andern Gases verwandelt, so mufs letzteres offenbar aus $1 / 2$ Vol. Chlor und 1 Vol. Methyl minus $1 / 2$ Vol. Wasserstoff bestehen und sein specifisches Gewicht mufs $=2,226$ seyn :

\begin{tabular}{ccc}
1 Vol. Kohlendampf & 0,82922 \\
$2^{1 / 2} \pi$ Wassersioff & 0,17270 \\
$1 / 2 \pi$ & Chlor & 1,22445 \\
\hline Condensirt zu 1 Volumen & $2,22637$.
\end{tabular}

Diefs ist aber die Condensation und Zusammensetzung des Chloräthyls. Der Rechnung zu Folge erfordert 1 Vol. desselben 3,25 Vol. Sauerstoff zur Verbrennung und erzengt 2 Vol. Kohlensäure; die obige Analyse hat damit in hinlänglicher Uebereinstimmung 3,12 Vol. verbrannten Sauersloffs und 1,93 Vol. der gebildeten Kohlensäure auf 1 Vol. des brennbaren Gases ergeben.

Wenn gleich jenes Gas in seiner Zusammensetzung, der Condensation seiner Elemente und folglich seinem specifischen Gewichte mil dem Chloräthyl genau übereinstimmt, so ist es dennoch nicht derselbe Körper, sondern nur eine isomere Verbindung. Denn während das Chlorälhyl unter $+12^{\circ} \mathrm{C}$. liquid wird und bei $-18^{\circ} \mathrm{C}$. krystallisirl ${ }^{*}$ ), so behält jenes Gas, durch eine Kugel von phosphorsaurem Natron von Salzsäure befreit

*) I.̈̈wig, Chemic der org. Verbindungen 2. Aufl. Bd. II. S. 434. 
und durch eine Kalikugel entwässert, selbst bei $-18^{\circ} \mathrm{C}$. seinen Aggregatzustand unverändert bei. Auch unterscheiden sie sich, jedoch weniger bestimmt, durch ihre verschiedene Auflöslichlieit in Wasser, welches vom Chloräkhyl ein gleiches Volumen aufnimmt, von dem andern Gase aber bei einer Teinperatur von $+19^{\circ} \mathrm{C}$. und \% Almosphärendruck Wasser nahe sein zweifaches Volumen alısorbirt.

Es ist kaum zu bezweifeln, dafs jene bemerkenswerthe Isomerie in einer von der rationellen Zusammensetzung des Chloräthyls abweichenden Gruppirung der Alome ihren Grund hat. Vielleicht ist der neue gasförmige Körper eine gepaarte Verbindung des Methyls mit einem andern Atom Methyl, worin 1 Aeq. Wasserstoff durch Chlor vertreten wird $=\mathrm{C}_{2} \mathrm{H}_{3} \cdot \mathrm{C}_{2}\left(\begin{array}{l}\mathrm{H}_{2} \\ \mathrm{El}\end{array}\right)$. Ob und in wie weit dicse Hypothese richtig ist, inufs durch spätere Versuche ermiltelt werden.

Uebrigens zeigt jenes Gas manche Uebereinstimmung mit dem Chlorällyyl; es besitzt einen sehr verwandten Geruch, verbrennt wie jenes unter Bildung von Salzsäuredämpfen mit smaragdgrüner Flamme und wird von einem Uebermafs von Chlor im Sonnenlichte in einen festen, camphorartigen Körper verwandelt, welcher ohne Zweifel Chlorkohlenstoff $=\mathrm{C}_{2} \mathrm{Gl}_{3}$ ist. Wir haben aus Mangel an Material seine Eigenschaften nicht weiler sludirt.

\section{Kyanäthin.}

Wenn man von 1 Alom Cyanälhyl 1 Alom Methyl abzieht, so bleibt das Glied $\mathrm{C}_{2} \mathrm{H}_{2} \mathrm{~Gy}$ übrig, welches - sich offenbar in der zähen gelblichen Masse befinden mufs, die bei der Darstellung des Methyls an der Stelle des Kaliums zurückbleibt. Es ist uns leider bis jetzt nicht gelungen, zu ermilteln, welche Verbindung jener Körper, oder wénigstens die Gruppe $C_{2} \quad I_{2}$ eịngeht. Behandelt man nämlich den Rückstand mit Wasser, 
so erhält man in der Auflösung eine reichliche Menge Cyankalium und aufserdem eine weifse unlösliche Substanz, welche indessen, wie aus den nachfolgenden Beobachtungen hervorgeht, aufser allem Zusammenhange mit der gesuchten Verbindung steht.

Jene in Wasser unlösliche Substanz, welche wegen ilrer merkwürdigen Beziehungen zu dem Cyanäthyl unsere Aufmerksamkeit im hohen Grade in Anspruch nahm, wurde, durch Auswaschen mit kaltem Wasser von Cyankalium und Cyanäthyl befreit, in siedendem Wasser gelöst. Beim Erkalten setzten sich daraus kleine perlmulterglänzende Krystallblättchen ab, welche bei $100^{\circ}$ gelrocknet folgende Zusammenselzung zeigten. $0,3557 \mathrm{Grm}$. gaben mit Kupferoxyd verbrannt *) 0,856 Grm. Kohlensäure und 0,300 Grm. Wasser.

*) Bei dieser und den nachfolgenden Analysen wurde nach beendeter Verbrennung mit Kupferoxyd, durch Erhitzen von geschmolzenem überchlorsaurem Kali, welches sich im hinteren Theile der Röhre befand und durch einen ausgeglühten Asbestpfropfen von dem Kupferoxyd getrennt war, ein Strom von Sauerstoff uber das reducirte Kupfer geleitet.

Ueberchlorsaures Kali eignet sich dazu besser, als das chlorsaure Salz, weil bei ersterem die Gasentwickelung viel gleichmäfsiger und zu Anfang weniger stürmisch ist. Der Kaliapparat war aufserdem mit einem kleinen, Stũcke von geschmolzenem Kalihydrat enthaltenden, Röhrchen versehen, um die von den die Kalilauge dirchstreichenden Luftblasen fortgeführten Wasserdämple zu absorbircn. Beide wurden zusammen gewogen. Zuletzt wenn der Kaliapparat vom Chlorcalciumrohr getrennt war, wurde durch Saugen der im ersteren möglicher Weise noch enthaltene freie Sauerstoff durch atmosphärische Luft ersetzt.

Manche sehr kohlenstoffreiche Verbindungen lassen sich mit blofsem 'Kupferoxyd ohne Sauerstoffgas nie vollständig verbrennen. Benzol, Naphtalin und ähnliche flüchtige, kohlenstoffreiche Kòrper gaben, mit Kupferoxyd allein verbrannt, im Durchschnitt 1/2 pC. Kohlenstotf zu wenig, bei gleichzeitiger Anwendung von Saverstoff jedoch fast absolut genaue Resultate. Jener Verlust scheint durch die Bildung von Kohlenkupfer veraulaist zu werden. 
0,2055 Grm. gaben 0,495 Grm. Kohlensäure und 0,171 Grm. Wasser.

Die Bestimmung des. Stickstoffgehalts, nach Bunsens Methode durch Glühen einer unbestimmten Menge Substanz mit Kupferoxyd und metall. Kupfer in einer stickstofffreien evacuirten und darauf hermetisch versehlossenen Glasröhre ausgeführt, gab folgende Dalen :

beob. Vol. Temp. Quecksilber- Bar. Corr. Vol. b.

C. săule über d. $\quad 0^{\circ} \mathrm{C} . \mathrm{u}^{1} \mathbf{1}^{\mathrm{m}}$ Wanne Druck

Gesainmivolumen
$\mathrm{CO}_{2}+\mathrm{N}$ (feucht) $\quad\left\{185,1^{\circ} \quad 20,9 \quad 370,4 \mathrm{~mm} \quad 753,9 \mathrm{~mm} 62,81\right.$ Nach Absorption d:
$\mathrm{CO}_{2}$ (feucht)

Daraus ergiebt sich das Verhältnifs der Kohlensäure zum Stickstoff $=6: 1,009$.

Procentische Zusammensetzung : $(C=75 ; H=12,5)$.

\begin{tabular}{crrr} 
& berechnet & \multicolumn{2}{c}{ gefunden } \\
$\mathrm{C}_{6}$ & 65,5 & $\mathbf{6 5 , 6}$ & 65,6 \\
$\mathrm{H}_{3}$ & 9,1 & 9,3 & 9,2 \\
$\mathrm{~N}$ & 25,4 & 25,5, & 25,5 \\
\hline & 100,0 & 100,4 & $100,3$.
\end{tabular}

Die analysirte Verbindung hat demnach die Zusammensetzung des Cyanäthyls. Sie ist jedoch weit entfernt, irgend eine Eigenschaft mit jenem zu theilen. Șie löst sich in allen Säuren mit Leichtigkeit auf, giebt damit in Wasser und Alkohol lösliche, theilweise schön krystallisirende Salze und wird daraus durch Kali, Ammoniak und die kohlensauren Alkalien unverändert gefältt, kurz sie ist eine organische Basis.

Wir nennen sie Kyanäthin, um damit an ihre Abstammung vom Cyanälhyl zu erinnern. Die Formel des Cyanäthyls entspricht jedoch nicht der atomistischen Zusammensetzung des 
Kyanäthins, sondern mufs, wie sich aus der Analyse seiner Salze ergiebt, verdreifacht werden. Ein Alom dieser Basis besteht demnach aus : $\mathrm{C}_{18}, \mathrm{H}_{13} \mathrm{~N}_{s}$.

Das Kyanäthin ist in reinen Zustande eine weilse, geruchund fast ganz geschmacklose flüchtige Substanz, schmilzt bei ohngefähr $190^{\circ} \mathrm{O}$. und fängt nahe bei $280^{\circ} \mathrm{C}$. an zu sieden, wobei sie jedoch eine partielle Zersetzung erleidet. Sie ist in Alkohol in fast allen Verhältnissen löslich, im kalten Wasser sehr wenig, in kochendem Wasser leichter löslich und scheidet sich aus letzterem, wie erwälınt, beim langsamen Erkalten in kleinen irisirenden Krystallblättchen ab. Die warme wässrige Lösung zeigt eine schwache, aber deutlich alkalische Reaction. Die Basis kann anhaltend mil Kalilauge gekocht werden, ohne die Zerselzung des Cyanäthyls und überhaupt ohne eine Veränderung zu erleiden. Wird sie damit zur Trockne verdampft und im Silbertiegel zum Schmelzen erhitzt, so sublimirt der gröfste Theil derselben unverändert ab, olıne dafs der Rückstand geschwärzt wird.

Die Salze des Kyanäthins laben durcligehends einen bilterlichen herben Geschmack und sind alle in Wasser und Alkohol löslich.

Das salpetersaure Kyanäthin $=\begin{array}{lllll}\mathrm{C}_{13} & \mathrm{II}_{15} & \mathrm{~N}_{8} & \mathrm{HO}, \mathrm{NO}_{3}\end{array}$ durch Auflösung der Basis in verdünnter Salpetersäure erhalten, krystallisirt beim freiwilligen Verdunsten in grofsen farblosen Prismen; es reagirt nach dean Umkrystallisiren vollkommen neutral. Die Analyse des bei $100^{\circ} \mathrm{C}$. getrockneten Salzes hat tolgende Zalılen gegeben.

0,401 Grm. gaben 0,699 Grm. Kohlensäure und 0,256 Grm. Wasser.

Zwei nach Buñsens Methode ausgeführte Stickstoffbestimmungen lieferten folgende Resultate : 
des Cyanäthyls durch Einwoirlung von Kalìum. 285

I. beob. Vol. Temp. Quecksilber- Bar. Corr. Vol. b.

C. săule über d. $\quad 0^{\circ} \mathrm{C}$. u. $1 \mathrm{~m}$ Wanne Druck

$\left.\begin{array}{l}\text { Anfängl. Volumen } \\ \mathrm{CO}_{2}+\mathrm{N} \text { (feucht) }\end{array}\right\} 132,0 \quad 21,2^{\circ} \quad 134,6 \mathrm{~mm} \quad 746,9 \mathrm{~mm} \quad 72,74$ $\begin{aligned} & \begin{array}{l}\text { Nach Absorption } \\ \text { der } \mathrm{CO}_{2} \text { (feucht) }\end{array}\end{aligned} \mid \begin{array}{lllll}28,2 & 21,2^{\circ} & 236,8 & 746,9 & 13,30\end{array}$

II.

$\begin{aligned} & \text { Anfängl. Volumen } \\ & \mathrm{CO}_{2}+\mathrm{N} \text { (feucht) }\end{aligned} \mid 134,7 \quad 18,4^{\circ} \quad 130,0 \mathrm{~mm} \quad 745,3 \mathrm{~mm} \quad 75,76$

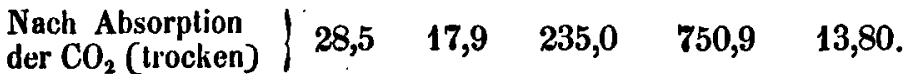

Daraus berechnet sich das Verhältnifs der Kohlensäure zum Stickstoff $=$

$$
\begin{array}{lll}
\text { (I) } 4,5: & 1,007 \text {. } \\
\text { (II) } 4,5: & 1,002 \text {. }
\end{array}
$$

Procentische Zusammensetzung :

\begin{tabular}{lcc} 
& berechnet & gefunden \\
$\mathbf{C}_{18}$ & $\mathbf{4 7 , 4}$ & $\mathbf{4 7 , 5}$ \\
$\mathbf{H}_{18}$ & $\mathbf{7 , 0}$ & $\mathbf{7 , 0}$ \\
$\mathrm{N}_{4}$ & $\mathbf{2 4 , 5}$ & $\mathbf{2 4 , 6}$ \\
$\mathbf{O}_{8}$ & $\mathbf{2 1 , 1}$ & $n$ \\
\hline \multicolumn{3}{c}{$100,0}$.
\end{tabular}

Das schwefelsaure und salzsaure Kyanäthin sind in Wasser sehr lösliche, nicht krystallisirbare Salze. Das essigsaure Salz verliert beim Abdampfen auch im Vacuum Essigsäure und verwandelt sich in eine unlösliche basische Verbindung. Das oxalsaure Salz, durch Neutralisation der Sãure mil einem Ueberschufs der Basis erhalten, giebt beim freiwilligen Verdunsten der filtrirten Lösung grofse, wohl gebildete, prismatische Krystalle. Auch das chlorkohlendithionsaure Salz hat grofse Neigung zu krystallisiren.

Wie das Ammoniak und die organischen Basen bildet das salzsaure Kyanälhin mit Platinchlorid ein Doppelsalz von aus- 
gezeichneter Schönheit, welches sich in Form eines gelblich rothen krystallinischen Niederschlags abscheidet, wenn concentrirte Lösungen beider vermischt werden. Es ist in Alkohol, auch in einer Mischung von Alkohol und Aether ziemlich leicht, in Wasser schwieriger löslich und krystallisirt daraus beim langsamen Verdunsten in grofsen rubinrothen Octaedern. Die alkoholische Auflösung erleidet beim Kochen eine Zersetzung, in Folge deren sich Chlorplatinammonium bildet.

Der obige Niederschlag mit kallem Wasser einige Male ausgewaschen, darauf zwischen Filtrirpapier geprefst und bei $100^{\circ}$ getrocknet, gab bei der Analyse folgende Zalılen :

0,797 Grm. in einem Porcellantiegel mit Alkohol befeuchtel und nach dessen Verbrennung anhaltend stark geglüht, lieferten 0,209 Grm. Platin.

0,427 Grm. gaben 0,456 Grm. Kohlensäure und 0,169 Grm. Wasser.

$$
\begin{aligned}
& \text { Zusammensetzung : } \mathrm{PlGi} \mathrm{H}_{2}+\mathrm{C}_{18} \mathrm{H}_{15} \mathrm{~N}_{3} \text {, HCI. } \\
& \text { berechnet gefunden } \\
& C_{1 x} \quad 29,1 \quad 29,1 \\
& H_{16} \quad 4,3 \quad 4,4 \\
& i_{3} \quad 11,4 \quad \text { \% } \\
& \mathrm{Fil}_{8} \quad 28,7 \quad \text { \% } \\
& \text { Pt } \frac{26,5 \quad 26,2}{100,0 .}
\end{aligned}
$$

Wir haben uns vergebens bemüht, die Umstände zu ermilleln, welche die merkwürdige Unsetzung der Elemente des Cyanäthyls zu Kyanälhin bedingen. Alle Versuche, letzteres auf einem andern Wege, als dem bezeichneten, darzustellen, sind fruchtlos geblieben. Selbst als wir das obige Verfahren dahin abänderten, dafs wir, anstatt das Cyanäthyl tropfenweise auf das Kalium fallen $\mathrm{zu}$ lassen, letzteres in Cyanäthyl warfen, 
bildete sich zwar Methylgas und Cyankalium, aber kaum eine Spur jener Basis. Cyanäthyl erleidet eben so wenig die gehoffte Veränderung, wenn man es in einer hermetisch verschlossenen starken Glasröhre für sich oder mit trocknem Cyankalium gemengt bis $\mathrm{zu} 240^{\circ} \mathrm{C}$. erhitzt; es bleibt dabei völlig unverändert. Uebrigens ist auch die nach dem angegebenen Verfahren erhaltene Menge der Basis sehr gering, sie beträgt nur wenige Procente vom angewandten Cyanälhyl.

Es ist schwer, sich von der rationellen Zusammensetzung des Kyanäthins einen Begriff $\mathrm{zu}$ machen. Um darüber eine Hypothese aufzustellen, welche es dem Anilin anreihet, der wir jedoch gegenwärtig nicht den mindesten Werth beilegen, so kann man sich vorstellen, dafs es gleich jenem ein gepaartes Amid sey, in dessen Paarling zwei Aeq. Wasserstoff durch zwei Aeq. Cyan vertreten sind $\left.=\mathrm{C}_{14} \mid \begin{array}{l}\mathrm{H}_{13} \\ \mathrm{~Gy}_{2}\end{array}\right\} . \mathrm{NH}_{2}$. Ueber den Werth oder Unwerth dieser Ansicht müssen künftige Beobachlungen entscheiden.

Die vorstehenden Versuche sind im Laboratorium des Hrn. Prof. Bunsen angestellt, welcher uns während unseres Aufenthalts in Marburg die Benutzung desselben freundschaftlichst gestattete. Mōge es uns erlaubt seyn, ihm hier für die vielfältigen Beweise seiner Güte und seines Wohlwollens unsern wärmsten Dank abzustatten. 